\title{
Compactifications of $\mathbb{N}$ and Polishable subgroups of $S_{\infty}$
}

\author{
by
}

Todor Tsankov (Pasadena, CA)

\begin{abstract}
We study homeomorphism groups of metrizable compactifications of $\mathbb{N}$. All of those groups can be represented as almost zero-dimensional Polishable subgroups of the group $S_{\infty}$. As a corollary, we show that all Polish groups are continuous homomorphic images of almost zero-dimensional Polishable subgroups of $S_{\infty}$. We prove a sufficient condition for these groups to be one-dimensional and also study their descriptive complexity. In the last section we associate with every Polishable ideal on $\mathbb{N}$ a certain Polishable subgroup of $S_{\infty}$ which shares its topological dimension and descriptive complexity.
\end{abstract}

1. Introduction. It is well known that every compact metrizable topological space $X$ can be realized in a unique way as the remainder $\widetilde{X} \backslash \mathbb{N}$ of a metrizable compactification $\widetilde{X}$ of the countable discrete space of the natural numbers $\mathbb{N}$ (see Propositions 2.1 and 2.3). This allows us to associate with each compact metrizable $X$ the homeomorphism group $\mathcal{H}(\widetilde{X})$ and a certain subgroup of it, called the structure group of $X$ (see Definition 2.5 below). These groups were first studied by Lorch [8], who proved the following interesting result:

THEOREM 1.1 (Lorch). Two compact metrizable spaces are homeomorphic if and only if their structure groups are isomorphic.

Both the group $\mathcal{H}(\widetilde{X})$ and the structure group of $X$ can be viewed as Polishable subgroups of $S_{\infty}$, the group of all permutations of $\mathbb{N}$ (see Proposition 2.4 below). We study the topological dimension of the Polish topologies of those groups as well as their descriptive complexity. In particular, we prove the following (see Theorem 3.1, Corollary 4.7, and Theorem 4.8 below):

2000 Mathematics Subject Classification: Primary 54H05, 54H15; Secondary 54F50.

Key words and phrases: almost zero-dimensional, Polishable ideals, compactifications, permutation groups, Polish groups.

Research was partially supported by NSF grant DMS-9987437. The author wishes to thank his advisor A. S. Kechris as well as S. Solecki and C. Rosendal for helpful discussions on the topic of this paper. Thanks are also due to H. Toruńczyk for valuable comments and to an anonymous referee for suggestions which significantly improved the presentation. 
TheOREM 1.2. The group $\mathcal{H}(\widetilde{X})$ is almost zero-dimensional (and thus at most one-dimensional). It is one-dimensional if the group $\mathcal{H}(X)$ contains a path of finite length (in the natural complete metric of the group). Both $\mathcal{H}(\widetilde{X})$ and the structure group of $X$ are $\Pi_{3}^{0}$ subgroups of $S_{\infty}$ and they are $\Pi_{3}^{0}$-complete iff $X$ is infinite.

As an interesting corollary of the construction, we show the following (see Corollary 4.11):

TheOREM 1.3. Every Polish group is a continuous homomorphic image of an almost zero-dimensional Polishable subgroup of $S_{\infty}$.

This is related to the open problem of whether every Polish group is a factor of a zero-dimensional Polish group.

In the last section of the paper we study Polishable ideals on $\mathbb{N}$ and certain almost zero-dimensional Polishable subgroups of $S_{\infty}$ associated with them.

Recall that a topological space is called Polish if it is separable and completely metrizable; a topological group is Polish if its topology is Polish. A Borel subgroup $H$ of a Polish group $G$ is called Polishable if there exists a Polish group topology on $H$ which has the same Borel structure as the one inherited from $G$. By $[5,9.10]$, the Polish topology of a Polishable $H$ is always finer than the inherited topology. Two examples of Polish groups are the homeomorphism groups of compact metrizable spaces with the compactopen topology, which coincides with the uniform convergence topology, and the group $S_{\infty}$ of permutations of the natural numbers with the pointwise convergence topology. A complete metric on $S_{\infty}$ is given by

$$
d(f, g)=2^{-\min \{f \neq g\}}+2^{-\min \left\{f^{-1} \neq g^{-1}\right\}} .
$$

The support of a permutation $f \in S_{\infty}$, denoted by $\operatorname{supp} f$, is the set of points moved by $f$. For a detailed treatment of Polish spaces and Polish and Polishable groups, the reader is referred to [5].

In any metric space we will denote by $B_{r}(x)$ the open ball with center $x$ and radius $r$. Since we will often work with different topologies on the same space, to avoid confusion, we will sometimes explicitly mention the topology, e.g., $(X, \tau)$ is the space $X$ with the topology $\tau$. Throughout this paper, $I$ denotes the unit interval $[0,1]$ and $Q=I^{\mathbb{N}}$ is the Hilbert cube.

2. Compact spaces as remainders of compactifications of $\mathbb{N}$. The following fact is well known; we include a simple proof, due to H. Toruńczyk, and note the effectiveness of the construction.

Proposition 2.1. For every compact metrizable space $X$, there exists a metrizable compactification $\widetilde{X}$ of $\mathbb{N}$ (taken with the discrete topology) such that the remainder $\widetilde{X} \backslash \mathbb{N}$ is homeomorphic to $X$. 
Proof. Fix a countable dense set $D=\left\{a_{k}\right\}$ in $X$. Set $\widetilde{X}=X \times\{0\} \cup A$, where $A=\bigcup\left\{a_{1}, \ldots, a_{n}\right\} \times\{1 / n\}$. Then $A$ is countable, discrete, and dense in $\tilde{X} ; \tilde{X}$ is compact as a closed subspace of $X \times[0,1]$.

We will think of the space $\widetilde{X}$ as the union $X \cup \mathbb{N}$ and we will also fix a compatible metric $d$ on $\widetilde{X}$. Consider the homeomorphism group $\mathcal{H}(\widetilde{X})$. With the topology induced by the metric

$$
\partial^{\prime}(f, g)=\sup _{x \in \widetilde{X}} d(f(x), g(x))
$$

it is a Polish group. The metric $\partial^{\prime}$ is not complete but it is equivalent to the complete metric $\partial$ defined by

$$
\partial(f, g)=\partial^{\prime}(f, g)+\partial^{\prime}\left(f^{-1}, g^{-1}\right) .
$$

Lemma 2.2. Let $f: X \rightarrow X$ be a homeomorphism and $\tilde{f}$ a homeomorphism of $\widetilde{X}$ such that $f$ and $\widetilde{f}$ agree on $X$. If $g: X \rightarrow X$ is another homeomorphism and $\partial(f, g)<r$, then there exists a homeomorphism $\widetilde{g}$ of $\widetilde{X}$ such that $\widetilde{g}$ extends $g$ and $\partial(\widetilde{f}, \widetilde{g})<r$.

Proof. Set $\varepsilon=(r-\partial(f, g)) / 6$. Since $\tilde{f}, g, \widetilde{f}^{-1}, g^{-1}$ are all uniformly continuous, we can find $\delta<\varepsilon$ so small that

$$
\begin{array}{ll}
\forall x, y \in \widetilde{X} & d(x, y)<\delta \Rightarrow d(\widetilde{f}(x), \widetilde{f}(y))<\varepsilon \quad \& \quad d\left(\widetilde{f}^{-1}(x), \widetilde{f}^{-1}(y)\right)<\varepsilon \\
\forall x, y \in X & d(x, y)<\delta \Rightarrow d(g(x), g(y))<\varepsilon \quad \& \quad d\left(g^{-1}(x), g^{-1}(y)\right)<\varepsilon .
\end{array}
$$

Using a standard back-and-forth argument, we will define a permutation $h: \mathbb{N} \rightarrow \mathbb{N}$ and then will set $\widetilde{g}=g \cup h$. First find a number $N$ so large that $d(n, X)<\delta$ for all $n>N$. Find points $x_{n} \in X$ such that $d\left(n, x_{n}\right)=d(n, X)$ for each $n$ and note that the set $\left\{x_{n}: n \in \mathbb{N}\right\}$ is dense in $X$. Set $h_{0}=$ $\left.\widetilde{f}\right|_{[0, N] \cup \widetilde{f}^{-1}([0, N])}$.

Now suppose we are at a forward step of the construction, say number $2 k-1$, and let $n=\min \left\{\mathbb{N} \backslash \operatorname{dom} h_{2 k-2}\right\}$. Find an $m$ such that $d\left(g\left(x_{n}\right), x_{m}\right)<$ $2^{-k} \delta$ and $m \notin \operatorname{ran} h_{2 k-2}$. Define $h_{2 k-1}$ to agree with $h_{2 k-2}$ on dom $h_{2 k-2}$ and set $h_{2 k-1}(n)=m$. Now we prove that this extension does not move us too far from $\widetilde{f}$. We have the following estimates:

$$
\begin{aligned}
d\left(h_{2 k-1}(n), \widetilde{f}(n)\right) \leq & d\left(m, x_{m}\right)+d\left(x_{m}, g\left(x_{n}\right)\right) \\
& +d\left(g\left(x_{n}\right), f\left(x_{n}\right)\right)+d\left(f\left(x_{n}\right), \widetilde{f}(n)\right) \\
\leq & d\left(g\left(x_{n}\right), f\left(x_{n}\right)\right)+3 \varepsilon \\
d\left(h_{2 k-1}^{-1}(m), \tilde{f}^{-1}(m)\right) \leq & d\left(\widetilde{f}^{-1}(m), f^{-1}\left(x_{m}\right)\right)+d\left(f^{-1}\left(x_{m}\right), g^{-1}\left(x_{m}\right)\right) \\
& +d\left(g^{-1}\left(x_{m}\right), x_{n}\right)+d\left(x_{n}, n\right) \\
\leq & d\left(f^{-1}\left(x_{m}\right), g^{-1}\left(x_{m}\right)\right)+3 \varepsilon .
\end{aligned}
$$


At a backward step $2 k$ proceed similarly, let $m=\min \left\{\mathbb{N} \backslash \operatorname{ran} h_{2 k-1}\right\}$. Find an $n$ such that $d\left(g\left(x_{n}\right), x_{m}\right)<2^{-k} \delta$ and $n \notin \operatorname{dom} h_{2 k-1}$. Define $h_{2 k}$ to agree with $h_{2 k-1}$ on $\operatorname{dom} h_{2 k-1}$ and set $h_{2 k}(n)=m$. Then (2) will again hold with $h_{2 k}$ replacing $h_{2 k-1}$.

Set $h=\bigcup_{k=0}^{\infty} h_{k}$ and $\widetilde{g}=g \cup h$. We will first prove that the $\widetilde{g}$ so defined is a homeomorphism of $\widetilde{X}$. It is enough to show that for any sequence $\left\{n_{k}\right\}$, $n_{k} \rightarrow z \Rightarrow h\left(n_{k}\right) \rightarrow g(z)$ for $z \in X$. This is easily seen:

$$
\begin{aligned}
n_{k} \rightarrow z & \Leftrightarrow x_{n_{k}} \rightarrow z \Leftrightarrow g\left(x_{n_{k}}\right) \rightarrow g(z) \\
& \Leftrightarrow x_{h\left(n_{k}\right)} \rightarrow g(z) \Leftrightarrow h\left(n_{k}\right) \rightarrow g(z) .
\end{aligned}
$$

Now, using (2), we also check that $\partial(\widetilde{f}, \widetilde{g})<r$ :

$$
\begin{aligned}
\partial(\widetilde{f}, \widetilde{g}) & =\sup _{x \in \widetilde{X}} d(\widetilde{f}(x), \widetilde{g}(x))+\sup _{x \in \widetilde{X}} d\left(\widetilde{f}^{-1}(x), \widetilde{g}^{-1}(x)\right) \\
& \leq \partial^{\prime}(f, g)+3 \varepsilon+\partial^{\prime}\left(f^{-1}, g^{-1}\right)+3 \varepsilon=\partial(f, g)+6 \varepsilon<r .
\end{aligned}
$$

The next proposition is essentially contained in [8]. It also follows from the proof of Lemma 2.2 above.

Proposition 2.3. If $X, Y$ are compact metrizable, $f: X \rightarrow Y$ is a homeomorphism, $\widetilde{X}$ and $\widetilde{Y}$ are compactifications of $\mathbb{N}$ as above, and $X \hookrightarrow \widetilde{X}$ and $Y \hookrightarrow \widetilde{Y}$ are given embeddings onto the remainders, then there exists a homeomorphism $\widetilde{f}: \widetilde{X} \rightarrow \widetilde{Y}$ such that the diagram

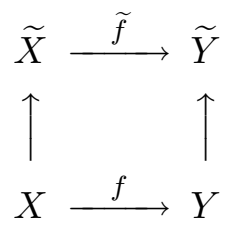

commutes.

In particular, Propositions 2.1 and 2.3 show that with every compact metrizable space we can associate a unique metrizable compactification $\widetilde{X}$ of $\mathbb{N}$ such that $X \cong \widetilde{X} \backslash \mathbb{N}$.

Proposition 2.4. The Polish group $\mathcal{H}(\widetilde{X})$ can be identified with a Polishable subgroup of $S_{\infty}$.

Proof. Since $\mathbb{N}$ is the set of all isolated points in $\tilde{X}$, for every homeomorphism $f \in \mathcal{H}(\widetilde{X})$ we must have $f(\mathbb{N})=\mathbb{N}$ and $f(X)=X$. Therefore the restriction map $R: \mathcal{H}(\widetilde{X}) \rightarrow S_{\infty}, R(f)=\left.f\right|_{\mathbb{N}}$, is a well defined group homomorphism. It is injective because $\mathbb{N}$ is dense in $\widetilde{X}$, and hence a homeomorphism is entirely determined by its action on $\mathbb{N}$. The map $R$ is also continuous as the composition of the identity map from $\mathcal{H}(\widetilde{X})$ to the same space, equipped with the pointwise convergence topology, and the restriction from the latter to $S_{\infty}$ (which also carries the pointwise convergence 
topology). Therefore we can identify $\mathcal{H}(\widetilde{X})$ with a Borel subgroup of $S_{\infty}$, which is Polishable because $\mathcal{H}(\widetilde{X})$ itself is Polish.

Now, following Lorch, we consider the pointwise stabilizer of $X$ in $\mathcal{H}(\widetilde{X})$.

Definition 2.5 (Lorch). The closed subgroup

$$
H(X)=\{f \in \mathcal{H}(\widetilde{X}): \forall x \in X \quad f(x)=x\}
$$

of $\mathcal{H}(\tilde{X})$ is called the structure group of $X$.

We will write $H$ instead of $H(X)$ if there is no danger of confusion. The restriction map $q: \mathcal{H}(\widetilde{X}) \rightarrow \mathcal{H}(X), q(f)=\left.f\right|_{X}$, is a continuous group homomorphism, has kernel $H$ and, by Proposition 2.3, is onto $\mathcal{H}(X)$. Therefore $H$ is a closed normal subgroup of $\mathcal{H}(\widetilde{X})$, and $\mathcal{H}(X) \cong \mathcal{H}(\widetilde{X}) / H$ as topological groups.

The restriction of the metric $d$ to $\mathbb{N}$ is totally bounded and induces the discrete topology on $\mathbb{N}$. Let $\mathcal{U H}(\mathbb{N}, d)$ denote the group of all uniform homeomorphisms of $\mathbb{N}$ with respect to the metric $d$ (i.e., all uniformly continuous permutations $\mathbb{N} \rightarrow \mathbb{N}$ with uniformly continuous inverses). $\mathcal{U} \mathcal{H}(\mathbb{N}, d)$ becomes a topological group with the uniform convergence topology. It is clear that any $f \in \mathcal{U H}(\mathbb{N}, d)$ extends to a homeomorphism $\widetilde{f}$ of $\widetilde{X}$, and conversely, any homeomorphism of $\widetilde{X}$ restricts to a uniform homeomorphism of $\mathbb{N}$. It is easy to check that this correspondence is a topological group isomorphism between $\mathcal{H}(\widetilde{X})$ and $\mathcal{U H}(\mathbb{N}, d)$, so from now on we can identify these two groups.

As was pointed out by A. S. Kechris, this viewpoint may also be relevant to the problem of characterizing the complexity of homeomorphism of compact metrizable spaces. More precisely, in view of the universality of the Hilbert cube $Q$ (cf. $[5,4.14]$ ), we can think of the hyperspace $K(Q)$ of all compact subsets of $Q$ (equipped with the Vietoris topology) as the space of all compact metrizable spaces and define the equivalence relation $E_{h}$ on $K(Q)$ by

$$
K E_{h} L \Leftrightarrow K \text { is homeomorphic to } L \text {. }
$$

Similarly, we can consider the Borel set $D \subseteq I^{\mathbb{N} \times \mathbb{N}}$ consisting of all totally bounded, discrete metrics on $\mathbb{N}$ of diameter not greater than 1 and define the equivalence relation $E_{u}$ on $D$ by

$$
d_{1} E_{u} d_{2} \Leftrightarrow\left(\mathbb{N}, d_{1}\right) \text { and }\left(\mathbb{N}, d_{2}\right) \text { are uniformly homeomorphic. }
$$

For two equivalence relations $E$ and $F$ defined on the standard Borel spaces $X$ and $Y$, respectively, we write $E \leq_{\mathrm{B}} F$ if there exists a Borel map $f: X \rightarrow Y$ satisfying

$$
x E y \Leftrightarrow f(x) F f(y) .
$$


If $E \leq_{\mathrm{B}} F$ and $F \leq_{\mathrm{B}} E$, we say that $E$ and $F$ are Borel bireducible. See [6] and the references therein for general background on the theory of equivalence relations and [4, Chapter 10] for more details on different (open) classification problems.

We have the following corollary from Propositions 2.1 and 2.3:

Corollary 2.6. $E_{h}$ and $E_{u}$ are Borel bireducible.

Proof. First construct a map $K(Q) \rightarrow D$ which reduces $E_{h}$ to $E_{u}$. Given $K \in K(Q)$, by $[5,12.13]$, we can find in a Borel way a dense countable subset of $K$ and then use the construction from the proof of Proposition 2.1 to define a metric on $\mathbb{N}$. Proposition 2.3 shows that this map is indeed a reduction.

Conversely, to reduce $E_{u}$ to $E_{h}$, consider first the inclusion map $i: D \rightarrow$ $Q^{\mathbb{N}}=I^{\mathbb{N} \times \mathbb{N}}$. By the proof of $[5,4.14]$ and using the total boundedness of the elements of $D$, if we consider $i(d), d \in D$, as a countable subset of $Q$, then the closure of $i(d)$ in $Q$ is homeomorphic to the completion of $(\mathbb{N}, d)$. The closure map $c: Q^{\mathbb{N}} \rightarrow K(Q)$ defined by $c\left(\left(a_{n}\right)\right)=\overline{\left\{a_{n}: n \in \mathbb{N}\right\}}$ is Borel and the composition $c \circ i$ is the desired reduction.

3. Descriptive complexity of $\mathcal{H}(\tilde{X})$ and $H(X)$. Both $\mathcal{H}(\tilde{X})$ and $H(X)$ are Borel subgroups of $S_{\infty}$, so we can ask where they fit in the Borel hierarchy.

Theorem 3.1. If $X$ is a one-point space, then $\mathcal{H}(\widetilde{X})=H(X)=S_{\infty}$. If $X$ has more than one but finitely many points, both $\mathcal{H}(\tilde{X})$ and $H(X)$ are $\boldsymbol{\Sigma}_{2}^{0}$-complete. Finally, if $X$ is infinite, both $\mathcal{H}(\widetilde{X})$ and $H(X)$ are $\boldsymbol{\Pi}_{3}^{0}$ complete.

Proof. Put $G=\mathcal{H}(\widetilde{X})$ and $H=H(X)$. The first statement of the theorem is obvious. Let now $X=\left\{x_{i}\right\}_{i=0}^{k}$ be finite and, without loss of generality, assume that $d\left(x_{i}, x_{j}\right)=1$ for $i \neq j$. Then $\boldsymbol{\Sigma}_{2}^{0}$ descriptions of $G$ and $H$ are given by:

$$
\begin{aligned}
& f \in G \Leftrightarrow \exists \sigma \in S_{k+1} \exists \delta \forall m \in \mathbb{N} d\left(m, x_{i}\right)<\delta \Rightarrow d\left(f(m), x_{\sigma(i)}\right)<1 / 2, \\
& f \in H \Leftrightarrow \forall i \leq k \exists \delta \forall m \in \mathbb{N} d\left(m, x_{i}\right)<\delta \Rightarrow d\left(f(m), x_{i}\right)<1 / 2 .
\end{aligned}
$$

Neither $G$ nor $H$ can be $G_{\delta}$ because they both contain the permutations with finite support, which are dense in $S_{\infty}$ (see Exercise 9.11 in [5]).

Let finally $X$ be infinite and $\left\{a_{k}\right\}_{k=0}^{\infty}$ be a countable dense set. First of all, the following are $\boldsymbol{\Pi}_{3}^{0}$ descriptions of $G$ and $H$ :

$$
\begin{aligned}
f \in G & \Leftrightarrow \forall \varepsilon \exists \delta \forall m, n \in \mathbb{N} d(m, n)<\delta \Rightarrow d(f(m), f(n))<\varepsilon, \\
f \in H & \Leftrightarrow f \in G \&\left(\forall \varepsilon \exists \delta \forall k \forall m \in \mathbb{N} d\left(m, a_{k}\right)<\delta \Rightarrow d\left(f(m), a_{k}\right)<\varepsilon\right) .
\end{aligned}
$$


Now consider the $\Pi_{3}^{0}$-complete set $C \subseteq 2^{\mathbb{N} \times \mathbb{N}}$ defined by

$$
A \in C \Leftrightarrow \forall n\{k:(n, k) \in A\} \text { is finite. }
$$

(We look at the elements of $2^{\mathbb{N} \times \mathbb{N}}$ as subsets of $\mathbb{N} \times \mathbb{N}$. For more information on $\Pi_{3}^{0}$-complete sets, see $[5,23 . \mathrm{A}]$.) We will construct a continuous map $\Phi: 2^{\mathbb{N} \times \mathbb{N}} \rightarrow S_{\infty}$ such that

$$
(A \in C \Rightarrow \Phi(A) \in H) \&(A \notin C \Rightarrow \Phi(A) \notin G),
$$

so $\Phi$ is a reduction of $C$ to both $G$ and $H$. Fix a convergent sequence $\left\{x_{k}\right\}_{k=0}^{\infty}$ of distinct elements of $X, x_{k} \rightarrow y$, and let $\left\{b_{k, j}\right\}$ be a 2-indexed sequence of distinct elements of $\mathbb{N}$ satisfying the following conditions:

(i) $\forall k, j \quad d\left(b_{k, j}, x_{k}\right)<2^{-(k+j)}$,

(ii) $\mathbb{N} \backslash\left\{b_{k, j}: k, j \in \mathbb{N}\right\}$ is dense in $X$.

Note that a sequence $\left\{b_{k_{n}, j_{n}}\right\}_{n=0}^{\infty}$ converges to a point of $X$ iff either $k_{n} \rightarrow \infty$ (in which case $b_{k_{n}, j_{n}} \rightarrow y$ ), or $k_{n}$ is eventually constant and $j_{n} \rightarrow \infty$ (then $\left.b_{k_{n}, j_{n}} \rightarrow x_{\lim k_{n}}\right)$. Now define $\Phi(A)=\prod_{(k, j) \in A}\left(b_{2 k, j} b_{2 k+1, j}\right)$, where $(m n)$ denotes the transposition in $S_{\infty}$ which switches $m$ and $n$. If $A \in C$, then the only limit point of $\operatorname{supp} \Phi(A)$ is $y$ and it is easy to see that $\Phi(A) \cup \operatorname{id}_{X}$ is a homeomorphism of $\tilde{X}$. If, on the other hand, $A \notin C$, then any continuous extension of $\Phi(A)$ to $X$ must switch $x_{2 k}$ and $x_{2 k+1}$ for some $k$, which is impossible because of (ii). Hence $\Phi(A) \notin G$ and (3) is verified.

4. Topological properties of the groups $\mathcal{H}(\tilde{X})$ and $H(X)$. On the groups $\mathcal{H}(\widetilde{X})$ and $H(X)$ we have two natural topologies, the Polish topology $\tau$ and the topology $\sigma$ inherited from $S_{\infty}$, i.e., the topology of pointwise convergence on $\mathbb{N}$. Clearly $\sigma \subseteq \tau$. We have the following easy fact.

Proposition 4.1. $(H, \tau)$ is zero-dimensional.

Proof. Note first that if $f, g \in H$ then there exists $a \in \mathbb{N}$ such that $\partial^{\prime}(f, g)=d(f(a), g(a))$. We will now show that every open ball $B_{r}\left(1_{H}\right)$ in $H$ is also closed. Indeed, let $\left\{g_{n}\right\}$ be a sequence in $B_{r}(1)$ such that $g_{n} \rightarrow$ $g \in H$. There exists $a \in \mathbb{N}$ for which $\partial^{\prime}\left(1_{H}, g\right)=d(a, g(a))$ but for some $n$, $g_{n}(a)=g(a)$ (because convergence in the topology of $H$ implies convergence in the coarser topology of $\left.S_{\infty}\right)$. Therefore, for this $n$,

$$
\partial^{\prime}\left(1_{H}, g\right)=d\left(a, g_{n}(a)\right) \leq \partial^{\prime}\left(1_{H}, g_{n}\right)<r .
$$

Hence $g \in B_{r}\left(1_{H}\right)$ and the proof is complete.

To continue our analysis, we need the notion of almost zero-dimensionality, introduced in Oversteegen-Tymchatyn [10]. Recall that a basis for a topological space $X$ is a collection $\mathcal{B}$ of (not necessarily open) subsets of $X$ such that for every open $U \subseteq X$ and every $x \in U$, there exists $B \in \mathcal{B}$ with $B \subseteq U$ and $x$ contained in the interior of $B$. Similarly, we say that $\mathcal{B}$ is a 
neighborhood basis at the point $x$ if for every open $U$ containing $x$, there exists $B \in \mathcal{B}$ with $B \subseteq U$ and $x$ contained in the interior of $B$. An open basis is a basis consisting of open sets.

Definition $4.2([10,2])$. A separable metrizable space is almost zerodimensional if there exists a basis for its topology consisting of intersections of clopen sets.

Note that almost zero-dimensionality is a hereditary property. An important fact about almost zero-dimensional spaces is the following:

Theorem 4.3 (Oversteegen-Tymchatyn [10], cf. Levin-Pol [7]). Every almost zero-dimensional space is at most one-dimensional.

The original definition of almost zero-dimensionality Oversteegen and Tymchatyn used to prove their theorem is somewhat different from Definition 4.2 (which we borrowed from Dijkstra-van Mill-Steprāns [2]) but the equivalence of the two definitions is proved in [2]. Almost zero-dimensional topologies are intimately related to certain coarser zero-dimensional topologies on the same space. This was noticed by van Mill and Dijkstra who suggested the following

Definition 4.4. Let $(X, \mathcal{T})$ be a separable metrizable space. We say that a separable metrizable zero-dimensional topology $\mathcal{W}$ on $X$ witnesses the almost zero-dimensionality of $(X, \mathcal{T})$ if $\mathcal{W} \subseteq \mathcal{T}$ and $(X, \mathcal{T})$ has a basis consisting of closed sets of $\mathcal{W}$.

As S. Solecki pointed out, using a result of his, we can exactly determine when the topology of a zero-dimensional Polish group witnesses the almost zero-dimensionality of a Polishable subgroup. To do this, we shall need some of the machinery developed in Solecki [13].

Let $(H, \tau)$ be a Polishable subgroup of a Polish group $(G, \sigma)$ and $\left\{V_{n}\right.$ : $n \in \mathbb{N}\}$ be an open neighborhood basis at 1 for $(H, \tau)$, satisfying the conditions

$$
V_{n}=V_{n}^{-1} \quad \text { and } \quad V_{n+1}^{3} \subseteq V_{n} .
$$

Let $F_{n}=\bar{V}_{n}^{\sigma}$ and for $x, y \in G$, define

$$
\delta_{l}(x, y)=\inf \left\{2^{-k}: x^{-1} y \in F_{k}\right\}, \quad \delta_{r}(x, y)=\inf \left\{2^{-k}: x y^{-1} \in F_{k}\right\},
$$

and

$$
d_{l}(x, y)=\inf \left\{\sum_{i=0}^{n-1} \delta_{l}\left(x_{i}, x_{i+1}\right): x_{0}=x, x_{n}=y, x_{i} \in G\right\}
$$

and similarly for $d_{r}$. Then

$$
\begin{aligned}
\widetilde{H}=\{g \in G: \forall V((1 & \in V \& V \text { is } \tau \text {-open }) \\
& \left.\left.\Rightarrow \exists h_{1}, h_{2} \in H \quad g \in{\overline{h_{1} V}}^{\sigma} \cap{\overline{V h_{2}}}^{\sigma}\right)\right\}
\end{aligned}
$$


is a $\Pi_{3}^{0}$ Polishable subgroup of $G$ with a Polish topology $\widetilde{\tau}$ defined by the metric $\varrho=d_{l}+d_{r}$ restricted to $\widetilde{H}$. Furthermore,

$$
\delta_{l} \geq d_{l} \geq \frac{1}{2} \delta_{l} \quad \text { and } \quad \delta_{r} \geq d_{r} \geq \frac{1}{2} \delta_{r} .
$$

$H$ is a dense subgroup of $(\widetilde{H}, \widetilde{\tau})$ and for any $\Pi_{3}^{0}$ set $A \subseteq G$ with $H \subseteq A$, $A \cap \widetilde{H}$ is comeager in $(\widetilde{H}, \widetilde{\tau})$. For all of the above, see [13].

LEMMA 4.5. Let $\left\{B_{n}\right\}$ be an arbitrary basis at 1 for $(H, \tau)$. Then $\widetilde{B}_{n}=$ $\bar{B}_{n}^{\sigma} \cap \widetilde{H}$ defines a basis at 1 for $\widetilde{H}$.

Proof. For each $k \in \mathbb{N}$, let $\widetilde{U}_{k} \subseteq \widetilde{H}$ be the open ball (in the metric $\varrho$ ) with center 1 and radius $2^{-k}$. Fix $k$ and find $n$ such that $B_{n} \subseteq V_{k+2}$. Then $\widetilde{B}_{n} \subseteq F_{k+2}$ and for any $x \in \widetilde{B}_{n}$,

$$
d_{l}(1, x) \leq \delta_{l}(1, x) \leq 2^{-(k+2)}<2^{-(k+1)}
$$

and similarly $d_{r}(1, x)<2^{-(k+1)}$. Hence $\varrho(1, x)<2^{-k}$ and $\widetilde{B}_{n} \subseteq \widetilde{U}_{k}$.

Conversely, for a fixed $n$, find $k$ such that $V_{k} \subseteq B_{n}$. Then for any $x \in$ $\widetilde{U}_{k+1}$,

$$
\delta_{l}(1, x) \leq 2 d_{l}(1, x) \leq 2 \varrho(1, x)<2^{-k} .
$$

Hence $x \in F_{k} \cap \widetilde{H} \subseteq \widetilde{B}_{n}, \widetilde{U}_{k+1} \subseteq \widetilde{B}_{n}$ and we are done.

Proposition 4.6. Let $(G, \sigma)$ be a zero-dimensional Polish group and $(H, \tau)$ a Polishable subgroup. Then the following are equivalent:

(i) $H$ is $\Pi_{3}^{0}$ in $G$;

(ii) $\left.\sigma\right|_{H}$ witnesses the almost zero-dimensionality of $(H, \tau)$;

(iii) every open set in $(H, \tau)$ is $\boldsymbol{\Sigma}_{2}^{0}$ in $\left(H,\left.\sigma\right|_{H}\right)$.

Proof. (i) $\Rightarrow\left(\right.$ ii). Let $(\widetilde{H}, \widetilde{\tau})$ be defined as in (5). Since $H$ is $\Pi_{3}^{0}$, by [13], $H$ is comeager in $\widetilde{H}$, so we must have $\widetilde{H}=H$ (see [5, Exercise 9.11]). Then the basis $\left\{\widetilde{B}_{n}\right\}$ of closed sets of $\left.\sigma\right|_{H}$, defined in Lemma 4.5 (starting with an arbitrary basis $\left\{B_{n}\right\}$ of $H$ ), shows that (ii) is true.

(ii) $\Rightarrow$ (iii). Let $\mathcal{B}$ be a basis for $\tau$ consisting of closed sets in $\left.\sigma\right|_{H}$. Since $\tau$ is Lindelöf, every open set is a countable union of elements of $\mathcal{B}$ and thus $\Sigma_{2}^{0}\left(\left.\sigma\right|_{H}\right)$.

(iii) $\Rightarrow($ i). This follows easily from a result in Farah-Solecki [3]. For $A \subseteq$ $G$ and a $\tau$-open $V \subseteq H$, we define the Vaught transform $A^{\triangle V}$ as

$$
A^{\triangle V}=\{g \in G:\{h \in H: h g \in A\} \text { is non-meager in }(V, \tau)\} .
$$

We will use a claim from the proof of [3, Theorem 3.1].

Claim. For $A \subseteq G, A \in \Sigma_{2}^{0}(\sigma)$ and any $\tau$-open $U \subseteq H, A^{\triangle U} \cap \widetilde{H}$ is $\widetilde{\tau}$-open.

Let $V$ be any open $\tau$-neighborhood of 1 in $H$. Since $V \in \Sigma_{2}^{0}\left(\left.\sigma\right|_{H}\right)$, there exists $A \subseteq G$ such that $A \in \boldsymbol{\Sigma}_{2}^{0}(\sigma)$ and $A \cap H=V$. Then $1 \in A^{\triangle V}$ and 
by the Claim, $A^{\triangle V} \cap \widetilde{H}$ is $\widetilde{\tau}$-open. Therefore $A^{\triangle V} \cap H$ is $\left.\widetilde{\tau}\right|_{H \text {-open and it }}$ is not hard to check that $A^{\triangle V} \cap H \subseteq V^{-1} V$. For any $\tau$-open neighborhood $U$ of 1 , we can find $V$ as above with $V^{-1} V \subseteq U$. Furthermore, $(H, \tau)$ is a Polishable subgroup of $(\widetilde{H}, \widetilde{\tau})$ and by $[5,9.10],\left.\widetilde{\tau}\right|_{H} \subseteq \tau$. Thus the set $\left\{A^{\triangle V} \cap H: V\right.$ is a $\tau$-neighborhood of 1$\}$ (where $A$ depends on $V$ ) is a basis at 1 for $\tau$ consisting of $\left.\widetilde{\tau}\right|_{H}$-open sets and hence $\left.\widetilde{\tau}\right|_{H}=\tau$. Therefore $\left(H,\left.\widetilde{\tau}\right|_{H}\right)$ is a Polish subgroup of $\widetilde{H}$. Since $H$ is dense in $\widetilde{H}$, we must have $H=\widetilde{H}$ (see [5, Exercise 9.11]).

Now, going back to the group $\mathcal{H}(\tilde{X})$, by Theorem 3.1 , it is $\Pi_{3}^{0}$ in $S_{\infty}$, hence Proposition 4.6 applies and we have the following corollary.

Corollary 4.7. $(\mathcal{H}(\tilde{X}), \tau)$ is almost zero-dimensional.

$\mathcal{H}(\tilde{X})$ can be zero-dimensional, e.g., if $X$ is a one-point space, then $\mathcal{H}(\widetilde{X}) \cong S_{\infty}$. Below we give a sufficient condition for $(\mathcal{H}(\widetilde{X}), \tau)$ not to be zero-dimensional. Recall that the length of a path $f:[a, b] \rightarrow Y$ in a metric space $(Y, d)$ is defined as

$$
\operatorname{len}(f)=\sup \left\{\sum_{i=0}^{n-1} d\left(f\left(x_{i}\right), f\left(x_{i+1}\right)\right): a=x_{0}<x_{1}<\cdots<x_{n}=b\right\} .
$$

If $x, y \in[a, b]$ write len $(x, y)$ for the length of the path $\left.f\right|_{[x, y]}$.

TheOREM 4.8. If the group $\mathcal{H}(X)$ has the property that there exists a homeomorphism $g \neq \mathrm{id}_{X}$ which can be connected to $\mathrm{id}_{X}$ via a path of finite length (in the complete metric $\partial$ ), then $\mathcal{H}(\widetilde{X})$ is not zero-dimensional.

Proof. Put $G=\mathcal{H}(\widetilde{X}), K=\mathcal{H}(X)$ and let $f$ be a path of finite length defined on the unit interval $[0,1]$ with $f(0)=1_{K}$ and $f(1)=g \neq 1_{K}$. Set $r=\partial\left(1_{K}, g\right)$. The quotient map $q: G \rightarrow K$ is Lipschitz and by Lemma 2.2 it sends open balls to open balls of the same radius. Suppose that $G$ is zero-dimensional; then there exists a clopen set $U \subseteq B_{r}\left(1_{G}\right)$. Towards a contradiction, define inductively transfinite sequences $\left\{t_{\alpha}\right\},\left\{h_{\alpha}\right\}$ and $\left\{\widetilde{h}_{\alpha}\right\}$, $\alpha<\omega_{1}$, of elements of $[0,1], K$ and $G$, respectively, satisfying the following conditions:

- $f\left(t_{\alpha}\right)=h_{\alpha}=q\left(\widetilde{h}_{\alpha}\right) ; \widetilde{h}_{\alpha} \in U$;

- $\alpha<\beta \Rightarrow t_{\alpha}<t_{\beta}$

- $\partial\left(\widetilde{h}_{\alpha}, \widetilde{h}_{\beta}\right) \leq 2 \operatorname{len}\left(t_{\alpha}, t_{\beta}\right)$.

Set $t_{0}=0, h_{0}=1_{K}, \widetilde{h}_{0}=1_{G}$. Suppose that the sequences have been defined for $\alpha<\beta$. If $\beta=\gamma+1$ is a successor, find $\varepsilon$ with $0<\varepsilon<\partial\left(h_{\gamma}, g\right)$ such that $B_{\varepsilon}\left(\widetilde{h}_{\gamma}\right) \subseteq U$. Set $t_{\beta}=\sup \left\{t \in\left[t_{\gamma}, 1\right]: \partial\left(h_{\gamma}, f(t)\right)=\varepsilon / 2\right\}$ and $h_{\beta}=f\left(t_{\beta}\right)$. Using Lemma 2.2 , find $\widetilde{h}_{\beta} \in G$ satisfying $q\left(\widetilde{h}_{\beta}\right)=h_{\beta}, \partial\left(\widetilde{h}_{\gamma}, \widetilde{h}_{\beta}\right)<3 \varepsilon / 4$, and hence, $\widetilde{h}_{\beta} \in U$. Finally, to verify the third condition, notice that for any 
$\alpha<\beta$,

$$
\begin{aligned}
\partial\left(\widetilde{h}_{\alpha}, \widetilde{h}_{\beta}\right) & \leq \partial\left(\widetilde{h}_{\alpha}, \widetilde{h}_{\gamma}\right)+\partial\left(\widetilde{h}_{\gamma}, \widetilde{h}_{\beta}\right) \leq 2 \operatorname{len}\left(t_{\alpha}, t_{\gamma}\right)+3 \varepsilon / 4 \\
& <2 \operatorname{len}\left(t_{\alpha}, t_{\gamma}\right)+2 \partial\left(h_{\gamma}, h_{\beta}\right) \leq 2 \operatorname{len}\left(t_{\alpha}, t_{\beta}\right) .
\end{aligned}
$$

Now consider the case when $\beta<\omega_{1}$ is a limit ordinal. Since $\beta$ is countable, there exists an increasing sequence $\left\{\gamma_{n}\right\}$ with $\lim \gamma_{n}=\beta$. By compactness of $[0,1], t_{\gamma_{n}}$ converges. By the inductive hypothesis, $\sum_{n} \partial\left(\widetilde{h}_{\gamma_{n}}, \widetilde{h}_{\gamma_{n+1}}\right) \leq$ $2 \sum_{n} \operatorname{len}\left(t_{\gamma_{n}}, t_{\gamma_{n+1}}\right)<\infty$, so $\left\{\widetilde{h}_{\gamma_{n}}\right\}$ is Cauchy and therefore converges. Set $\widetilde{h}_{\beta}=\lim \widetilde{h}_{\gamma_{n}}, h_{\beta}=q\left(\widetilde{h}_{\beta}\right), t_{\beta}=\lim t_{\gamma_{n}}$. By continuity, $f\left(t_{\beta}\right)=h_{\beta}$ and $\widetilde{h}_{\beta} \in U$ because $U$ is closed. Now fix $\alpha<\beta$ and verify the last condition:

$$
\partial\left(\widetilde{h}_{\alpha}, \widetilde{h}_{\beta}\right)=\lim _{n \rightarrow \infty} \partial\left(\widetilde{h}_{\alpha}, \widetilde{h}_{\gamma_{n}}\right) \leq \sup _{n} 2 \operatorname{len}\left(t_{\alpha}, t_{\gamma_{n}}\right) \leq 2 \operatorname{len}\left(t_{\alpha}, t_{\beta}\right) .
$$

As a result of the construction, we obtain an order preserving embedding $\omega_{1} \rightarrow[0,1]$, which is clearly impossible.

Proposition 4.9. There exists a path $f:[1 / 2,3 / 4] \rightarrow \mathcal{H}(I)$ with $\mathrm{id}_{I}=$ $f(1 / 2) \neq f(3 / 4)$ and of finite length (in the complete metric $\partial$ ).

Proof. For each $t \in[1 / 2,3 / 4]$, consider the homeomorphism $f(t): I \rightarrow I$ which maps linearly $[0,1 / 2]$ onto $[0, t]$ and $[1 / 2,1]$ onto $[t, 1]$. It is easy to see that $\partial(f(t), f(s)) \leq 3|t-s|$, so we have our path.

Endow the Hilbert cube $Q=I^{\mathbb{N}}$ with its standard metric

$$
d\left(\left(x_{0}, x_{1}, \ldots\right),\left(y_{0}, y_{1}, \ldots\right)\right)=\sum_{n=0}^{\infty} 2^{-n}\left|x_{n}-y_{n}\right| .
$$

Then we have the following

Corollary 4.10. There is a path $f:[1 / 2,3 / 4] \rightarrow \mathcal{H}(Q)$ of finite length with $\operatorname{id}_{Q}=f(1 / 2) \neq f(3 / 4)$, and hence $\mathcal{H}(\widetilde{Q})$ is one-dimensional.

Proof. The map $i: \mathcal{H}(I) \rightarrow \mathcal{H}(Q)$ defined by

$$
i(h)\left(x_{0}, x_{1}, \ldots\right)=\left(h\left(x_{0}\right), x_{1}, \ldots\right)
$$

is an isometric embedding.

It is an open problem whether every Polish group is a homomorphic image of a zero-dimensional Polish group. However, we have the following interesting corollary, again pointed out by Kechris:

Corollary 4.11. Every Polish group is a factor of an almost zerodimensional Polishable subgroup of $S_{\infty}$.

Proof. Let $K$ be a Polish group. It is well known that $\mathcal{H}(Q)$ is a universal Polish group (see Uspenskiu [15]), hence there exists an embedding $i: K \rightarrow$ $\mathcal{H}(Q)$ onto a closed subgroup of $\mathcal{H}(Q)$. Let $q: \mathcal{H}(\widetilde{Q}) \rightarrow \mathcal{H}(Q)$ be the quotient map. Then $q^{-1}(i(K))$ is a closed subgroup of $\mathcal{H}(\widetilde{Q})$ and $q^{-1}(i(K)) / H \cong K$. 
REMARK. Corollary 4.11 is false if we restrict ourselves to closed subgroups of $S_{\infty}$. In fact, using the characterization that the closed subgroups of $S_{\infty}$ are exactly the Polish groups which admit a basis at the identity consisting of open subgroups (see Becker-Kechris [1, Theorem 1.5.1]), it is not hard to show that any factor of a closed subgroup of $S_{\infty}$ is isomorphic to a closed subgroup of $S_{\infty}$.

5. Polishable ideals on $\mathbb{N}$. Recall that an ideal on $\mathbb{N}$ is a collection of subsets of $\mathbb{N}$ closed under finite unions and taking subsets. To avoid trivialities, we will also assume that every ideal contains the ideal of finite sets Fin. An ideal is called Polishable if it is a Polishable subgroup of the Cantor group $2^{\mathbb{N}}$ (with symmetric difference as the group operation). A lower semicontinuous (or lsc) submeasure on $\mathbb{N}$ is a function $\phi: \mathcal{P}(\mathbb{N}) \rightarrow[0, \infty]$ satisfying

- $\phi(\emptyset)=0$

- $a \subseteq b \Rightarrow \phi(a) \leq \phi(b)$ for any $a, b \subseteq \mathbb{N}$;

- $\phi(a \cup b) \leq \phi(a)+\phi(b) ; \phi(\{n\})<\infty$ for $n \in \mathbb{N}$;

- $\phi\left(\bigcup_{k} a_{k}\right)=\lim _{k} \phi\left(a_{k}\right)$ whenever $a_{0} \subseteq a_{1} \subseteq \cdots$.

With every lsc submeasure we associate the following two ideals:

$$
\operatorname{Exh}(\phi)=\left\{a \subseteq \mathbb{N}: \lim _{n} \phi(a \backslash n)=0\right\}, \quad \operatorname{Fin}(\phi)=\{a \subseteq \mathbb{N}: \phi(a)<\infty\} .
$$

(As is customary, we identify the natural number $n$ with the set of its predecessors.) It is easy to see that $\operatorname{Exh}(\phi) \subseteq \operatorname{Fin}(\phi)$ and $\operatorname{Fin}(\phi)$ is $\boldsymbol{\Sigma}_{2}^{0}$, while $\operatorname{Exh}(\phi)$ is $\Pi_{3}^{0}$ in $2^{\mathbb{N}}$. Since the ideals $\operatorname{Exh}(\phi)$ and $\operatorname{Fin}(\phi)$ do not change if we replace $\phi$ with the submeasure $\phi^{\prime}$ defined by $\phi^{\prime}(a)=\phi(a)+\sum_{n \in a} 2^{-n}$, we can restrict our considerations to submeasures $\phi$ satisfying $\phi(\{n\})>0$ for all $n$. An ideal $I$ is called a $P$-ideal if for every sequence $\left\{a_{n}\right\}$ of elements of $I$ there exists $a \in I$ such that $a_{n} \backslash a$ is finite for all $n$. The following is a summary of the results of Solecki $[12,11]$ which we shall need.

Theorem 5.1 (Solecki). An ideal $I$ is an analytic P-ideal iff it is Polishable iff there exists a finite, lsc submeasure $\phi$ with $I=\operatorname{Exh}(\phi) . I$ is $\boldsymbol{\Sigma}_{2}^{0}$ Polishable iff there exists a lsc $\phi$ with $I=\operatorname{Exh}(\phi)=\operatorname{Fin}(\phi)$.

If $I=\operatorname{Exh}(\phi)$, then the Polish topology on $I$ is induced by the metric $d(a, b)=\phi(a \triangle b)$, where $\triangle$ denotes the operation of symmetric difference.

We say that two ideals $I$ and $J$ are isomorphic if there exists a permutation $f: \mathbb{N} \rightarrow \mathbb{N}$ such that $a \in I \Leftrightarrow f(a) \in J$. We denote the trivial ideal $\mathcal{P}(\mathbb{N})$ simply by $\mathbb{N}$. If $I$ and $J$ are ideals on $\mathbb{N}$ then $I \oplus J$ is the ideal on $\mathbb{N} \times 2$ defined by

$$
I \oplus J=\{a \times\{0\} \cup b \times\{1\}: a \in I \& b \in J\} .
$$


An ideal is a trivial modification of Fin if it is of the form $\{a: a \cap b$ is finite $\}$ for some $b \subseteq \mathbb{N}$. If an ideal $I$ is Polishable, we will denote the topological space $I$ with its Polish topology by $I^{\tau}$. Since every Polishable ideal is $\Pi_{3}^{0}$, Proposition 4.6 implies that $I^{\tau}$ is almost zero-dimensional, as witnessed by the topology inherited from the compact group $2^{\mathbb{N}}$. It is also easy to check that $(I \oplus J)^{\tau}$ is homeomorphic to $I^{\tau} \times J^{\tau}$.

We associate with each Polishable ideal $I$ the subgroup $S_{I} \leq S_{\infty}$ defined by

$$
S_{I}=\left\{f \in S_{\infty}: \operatorname{supp} f \in I\right\} .
$$

Below we will make use of a lemma which can be proved in the same way as the fact that all automorphisms of $S_{\infty}$ are inner. A very detailed exposition can be found in Lorch [9].

Lemma 5.2. If $G_{1}$ and $G_{2}$ are isomorphic subgroups of $S_{\infty}$, both containing all permutations with finite support, then they are conjugate, i.e., there exists $f \in S_{\infty}$ such that $G_{2}=f^{-1} G_{1} f$.

Theorem 5.3. With the above definition, $S_{I}$ is a Polishable subgroup of $S_{\infty}$ which is almost zero-dimensional in its Polish topology. It is zerodimensional iff $I^{\tau}$ is zero-dimensional. The Borel complexity of $S_{I}$ in $S_{\infty}$ and $I$ in $2^{\mathbb{N}}$ is the same. Furthermore, the groups $S_{I}$ and $S_{J}$ are isomorphic (algebraically) iff $I$ and $J$ are isomorphic ideals.

Proof. Let $\phi$ be a lsc submeasure such that $I=\operatorname{Exh}(\phi)$. The group $S_{I}$ acts on $I$ in a natural way: $g \cdot a=\{g(n): n \in a\}$. The first thing we will check is that this action is continuous in the second variable, i.e.,

$$
\forall g \in S_{I} \forall \varepsilon \exists \delta \forall a \in I \quad \phi(a)<\delta \Rightarrow \phi(g \cdot a)<\varepsilon .
$$

(Continuity at $\emptyset$ is sufficient because $g \cdot(a \triangle b)=(g \cdot a) \triangle(g \cdot b)$.) Fix $g \in S_{I}$ and $\varepsilon>0$. Find $N \in \mathbb{N}$ such that $\phi(\operatorname{supp} g \cap[N, \infty))<\varepsilon / 2$, and $\delta<\varepsilon / 2$ so small that $\phi(a)<\delta \Rightarrow a \cap g^{-1} \cdot[0, N)=\emptyset$. Now for any $a \in I$ with $\phi(a)<\delta$, we have

$$
\phi(g \cdot a) \leq \phi(a \cup(\operatorname{supp} g \cap[N, \infty))) \leq \phi(a)+\phi(\operatorname{supp} g \cap[N, \infty))<\varepsilon .
$$

Define the left invariant metric $\partial^{\prime}$ on $S_{I}$ by $\partial^{\prime}(f, g)=\phi(\{f \neq g\})$. It is clear that every open ball in this metric is Borel in $S_{\infty}$. We next check that multiplication is continuous. Fix $f_{0}, g_{0} \in S_{I}$ and $\varepsilon>0$. Using (7), find $\delta<\varepsilon / 2$ so small that $\phi(a)<\delta \Rightarrow \phi\left(g_{0}^{-1} \cdot a\right)<\varepsilon / 2$. Now for any $f, g \in S_{I}$ with $\max \left(\partial^{\prime}\left(g, g_{0}\right), \partial^{\prime}\left(f, f_{0}\right)\right)<\delta$, we have

$$
\begin{aligned}
\partial^{\prime}\left(f g, f_{0} g_{0}\right) & =\phi\left(\left\{f g \neq f_{0} g_{0}\right\}\right) \leq \phi\left(\left\{g \neq g_{0}\right\}\right)+\phi\left(\left\{f g_{0} \neq f_{0} g_{0}\right\}\right) \\
& =\partial^{\prime}\left(g, g_{0}\right)+\phi\left(g_{0}^{-1} \cdot\left\{f \neq f_{0}\right\}\right) \leq \varepsilon / 2+\varepsilon / 2=\varepsilon .
\end{aligned}
$$

The map $f \mapsto f^{-1}$ is continuous because the metric is left invariant and multiplication is continuous. The next thing we show is that the metric 
$\partial(f, g)=\partial^{\prime}(f, g)+\partial^{\prime}\left(f^{-1}, g^{-1}\right)$ is complete. Let $\left\{f_{n}\right\}$ be a Cauchy sequence in this metric. Without loss of generality, we can assume that $\phi(\{n\}) \geq 2^{-n}$, so $\partial$ dominates the standard complete metric on $S_{\infty}$ (1). Therefore the pointwise limit $g=\lim _{n} f_{n}$ exists. We check that $g \in S_{I}$. Fix $\varepsilon>0$ and $N \in \mathbb{N}$ such that $\phi\left(\left\{f_{m} \neq f_{n}\right\}\right)<\varepsilon / 4$ for all $m, n>N$. Fix $m>N$. Let $M \in \mathbb{N}$ be so large that $\phi\left(\operatorname{supp} f_{m} \backslash M\right)<\varepsilon / 4$. Suppose, towards a contradiction, that $\phi(\operatorname{supp} g \backslash M)>\varepsilon$. Then there is $M_{1}>M$ such that $\phi\left(\operatorname{supp} g \cap\left[M, M_{1}\right]\right)>\varepsilon / 2$. Find $k>N$ such that $f_{k}$ agrees with $g$ on $\left[0, M_{1}\right]$. Then $\phi\left(\operatorname{supp} f_{k} \backslash M\right)>\varepsilon / 2$, which contradicts the choice of $N$ and $M$. Therefore $\operatorname{supp} g \in \operatorname{Exh}(\phi)=I$. Now it remains to check that $\phi\left(\left\{f_{n} \neq g\right\}\right) \rightarrow 0$. Again fix an $\varepsilon$ and find $N$ such that $\phi\left(\left\{f_{m} \neq f_{n}\right\}\right)<\varepsilon / 2$ for all $m, n>N$. Fix $m>N$. Let $M$ be such that $\phi\left(\left\{f_{m} \neq g\right\} \backslash M\right)<\varepsilon / 2$. Find $n>m$ such that $f_{n}$ and $g$ agree on $M$. Then

$$
\phi\left(\left\{f_{m} \neq g\right\}\right) \leq \varepsilon / 2+\phi\left(\left\{f_{m} \neq g\right\} \cap M\right)=\varepsilon / 2+\phi\left(\left\{f_{m} \neq f_{n}\right\} \cap M\right)<\varepsilon .
$$

Finally, the topology defined by $\partial$ is separable because the group of permutations with finite support is dense in $S_{I}($ since $\phi(\operatorname{supp} f \backslash n) \rightarrow 0$ for all $f \in S_{I}$ and thus permutations in $S_{I}$ can be approximated in the metric $\partial^{\prime}$ by permutations of finite support). This completes the proof that $S_{I}$ is Polishable.

If $I=$ Fin or $I=\mathbb{N}$, the remaining statements are clear. Suppose now that this is not the case and let $b \notin I$ be an infinite set such that $\mathbb{N} \backslash b$ is infinite and in $I$. Fix a bijection $h$ between $b$ and $\mathbb{N} \backslash b$. Let $I^{\prime}=\left.I\right|_{b}=$ $\{a \cap b: a \in I\}=\operatorname{Exh}\left(\left.\phi\right|_{b}\right)$. Then $I^{\prime}$ is Polishable and $I \cong I^{\prime} \oplus \mathbb{N}$. Let $p: 2^{\mathbb{N}}=2^{b} \times 2^{\mathbb{N} \backslash b} \rightarrow 2^{b}$ be the projection and consider the continuous maps $\Phi: S_{\infty} \rightarrow 2^{\mathbb{N}}$ and $\Psi: 2^{b} \rightarrow S_{\infty}$ defined by

$$
\Phi(f)=\operatorname{supp} f \quad \text { and } \quad \Psi(a)=\prod_{n \in a}(n h(n)) .
$$

By the definition of $S_{I}, f \in S_{I} \Leftrightarrow \Phi(f) \in I$. Furthermore, for $a \in 2^{b}$, $\operatorname{supp} \Psi(a)=a \cup h(a)$ and hence

$$
a \in I \quad \Leftrightarrow \quad p(a) \in I^{\prime} \Leftrightarrow \Psi(p(a)) \in S_{I} .
$$

Those reductions prove the statement about the Borel complexity of $I$ and $S_{I}$. The fact that $S_{I}$ is $\Pi_{3}^{0}$, together with Proposition 4.6, implies that the Polish topology of $S_{I}$ is almost zero-dimensional.

Let now $I^{\tau}$ be zero-dimensional and $\left\{U_{k}\right\}$ be a clopen basis at $\emptyset$. Then $\left\{\Phi^{-1}\left(U_{k}\right)\right\}$ is a clopen basis for $S_{I}$ at 1 . Conversely, if $S_{I}$ is zero-dimensional, notice that $\Psi\left(I^{\prime}\right)=\Psi\left(2^{b}\right) \cap S_{I}$ is a closed subgroup of $S_{I}$ and hence the group homomorphism $\left.\Psi\right|_{I^{\prime}}: I^{\prime} \rightarrow S_{I}$ is a homeomorphic embedding $I^{\prime \tau} \hookrightarrow S_{I}$. Therefore $I^{\prime \tau}$ is zero-dimensional and since $I^{\tau} \cong I^{\prime \tau} \times 2^{\mathbb{N}}, I^{\tau}$ is also zerodimensional.

The last statement is a direct consequence of Lemma 5.2. 
Finally, we use our methods from the proof of Theorem 4.8 to sketch an alternative proof of the following fact, due to Solecki:

Proposition 5.4 (Solecki [14]). For a $\boldsymbol{\Sigma}_{2}^{0}$ Polishable ideal I, the following are equivalent:

(i) $I^{\tau}$ is zero-dimensional;

(ii) I is a trivial modification of Fin.

Proof. (ii) $\Rightarrow(\mathrm{i})$. Let $I=\{a: a \cap b$ is finite $\}$ for some $b \subseteq \mathbb{N}$. If $b$ is finite, then $I=\mathbb{N}$. If $b$ is co-finite, then $I=$ Fin. Finally, if $b$ is infinite and co-infinite, $I=\mathbb{N} \oplus$ Fin and $I^{\tau} \cong \operatorname{Fin}^{\tau} \times \mathbb{N}^{\tau} \cong \mathbb{N} \times 2^{\mathbb{N}}$ is zero-dimensional.

(i) $\Rightarrow$ (ii). Use Theorem 5.1 to find a lsc submeasure $\phi$ such that $I=$ $\operatorname{Exh}(\phi)=\operatorname{Fin}(\phi)$. Suppose, towards a contradiction, that $I^{\tau}$ is zero-dimensional but (ii) is not satisfied. Then it is not hard to see that

$$
\forall \varepsilon>0 \quad\{n: \phi(\{n\})<\varepsilon\} \notin I .
$$

Indeed, if not, find $\varepsilon>0$ with $\{n: \phi(\{n\})<\varepsilon\} \in I$ and set $b=\{n$ : $\phi(\{n\}) \geq \varepsilon\}$. Then $I=\{a: a \cap b$ is finite $\}$, a contradiction. Let $U \subseteq\{a$ : $\phi(a)<1\}$ be clopen. We will construct inductively a transfinite sequence $\left\{a_{\alpha}\right\}_{\alpha<\omega_{1}}$ of elements of $U$ satisfying $\alpha<\beta \Leftrightarrow a_{\alpha} \subsetneq a_{\beta}$, thus obtaining the desired contradiction. Start with $a_{0}=\emptyset$. At successor steps, given $a_{\beta}$, use the openness of $U$ and (8) to find $n \notin a_{\beta}$ such that $a_{\beta} \cup\{n\} \in U$ and set $a_{\beta+1}=a_{\beta} \cup\{n\}$. At a limit $\alpha$ set $a_{\alpha}=\bigcup_{\beta<\alpha} a_{\beta}$ and $\lim _{n} a_{\gamma_{n}}=a_{\alpha}$ in the Polish topology of $I$ for any sequence $\left\{\gamma_{n}\right\}$ cofinal in $\alpha$ (use $I=\operatorname{Exh}(\phi)$ here). Hence $a_{\alpha} \in U$.

\section{References}

[1] H. Becker and A. S. Kechris, The Descriptive Set Theory of Polish Group Actions, London Math. Soc. Lecture Note Ser. 232, Cambridge Univ. Press, Cambridge, 1996.

[2] J. J. Dijkstra, J. van Mill and J. Steprāns, Complete Erdös space is unstable, Math. Proc. Cambridge Philos. Soc. 137 (2004), 465-473,

[3] I. Farah and S. Solecki, Borel subgroups of Polish groups, Adv. Math. 199 (2006), 499-541.

[4] S. Gao and A. S. Kechris, On the classification of Polish metric spaces up to isometry, Mem. Amer. Math. Soc. 161 (2003), no. 766.

[5] A. S. Kechris, Classical Descriptive Set Theory, Grad. Texts in Math. 156, Springer, New York, 1995.

[6] -, New directions in descriptive set theory, Bull. Symbolic Logic 5 (1999), 161-174.

[7] M. Levin and R. Pol, A metric condition which implies dimension $\leq 1$, Proc. Amer. Math. Soc. 125 (1997), 269-273.

[8] E. R. Lorch, Certain compact spaces and their homeomorphism groups, in: Proc. International Conference on Partial Differential Equations Dedicated to Luigi Amerio on his 70th Birthday (Milan/Como, 1982), Rend. Sem. Mat. Fis. Milano 52 (1982), $75-86$ (1985). 
[9] E. R. Lorch, On the isomorphisms of certain groups of permutations of the integers, Appl. Anal. 15 (1983), 201-206.

[10] L. G. Oversteegen and E. D. Tymchatyn, On the dimension of certain totally disconnected spaces, Proc. Amer. Math. Soc. 122 (1994), 885-891.

[11] S. Solecki, Analytic ideals, Bull. Symbolic Logic 2 (1996), 339-348.

[12] -, Analytic ideals and their applications, Ann. Pure Appl. Logic 99 (1999), 51-72.

[13] —, Polish group topologies, in: Sets and Proofs (Leeds, 1997), London Math. Soc. Lecture Note Ser. 258, Cambridge Univ. Press, 1999, 339-364.

[14] - , Local inverses of Borel homomorphisms and analytic P-ideals, Abstr. Appl. Anal. 3 (2005), 207-220.

[15] V. Uspenskiŭ, A universal topological group with a countable basis, Funktsional. Anal. i Prilozhen. 20 (1986), no. 2, 86-87 (in Russian).

Department of Mathematics 253-37

California Institute of Technology

Pasadena, CA 91125, U.S.A.

E-mail: todor@caltech.edu

Received June 29, 2005;

in revised form January 14, 2006 